\title{
The Breakdown Process of Hexadecane Films Induced by Quasi-Static Loading"
}

\author{
Kazuyoshi MANABE $^{* *}$ and Ken NAKANO ${ }^{* *}$ \\ **Graduate School of Environment and Information Sciences, Yokohama National University, \\ 79-7 Tokiwadai, Hodogaya-ku, Yokohama-shi, Kanagawa, 240-8501 Japan \\ E-mail: kazu@davinci.jks.ynu.ac.jp
}

\begin{abstract}
A novel experimental method has been developed to quantify the breakdown processes of thin oil films induced by quasi-static loading. The method uses a steel-oil-mercury system along with complex impedance analysis, which provides the simultaneous measurement of the film thickness and breakdown ratio. In the case of hexadecane, the relationship between thickness and the breakdown ratio is represented by a single master curve independently of the indentation speed. Every breakdown process of hexadecane traces on the same master curve, and its final point is determined by the indentation load. The breakdown process of hexadecane includes two stages: one is the decrement of the film thickness without breakdown, and the other is the decrement of the film thickness with the drastic progress of breakdown.
\end{abstract}

Key words: Lubrication, Breakdown Process, Film Thickness, Breakdown Ratio, Steel-Oil-Mercury System, Complex Impedance Analysis

\section{Introduction}

Understanding the nature of lubricating films is essential for lubricated surfaces of machinery systems. In general, unbreakable lubricating films are required to prevent intimate contact between mating surfaces, which are produced by fluid film lubrication ${ }^{(1)}$. In practice, however, the transition from fluid film lubrication to boundary lubrication occurs inevitably with increasing load or decreasing relative velocity, which leads to an increase in the coefficient of friction. The Stribeck curve provides a qualitative explanation of the $\operatorname{transition}^{(2),(3)}$; however, its quantitative evaluation methods have not been established yet.

Therefore, Nakano and Akiyama ${ }^{(4)}$ have proposed a quantitative method for measuring the film thickness and breakdown ratio simultaneously, where the latter is defined as the ratio of the intimate contact area to the real contact area ${ }^{(5)}$. In order to avoid the difficulty of determining the real contact area, they use a steel-oil-mercury system, which means the system is expected to employ a single real contact by the fluidity of mercury. They have measured the complex impedance of the system and introduced a method for calculating the film thickness and breakdown ratio.

This paper describes the development of the method proposed by Nakano and Akiyama. The present study uses the steel-oil-mercury system, and its complex impedance is measured for quantifying the film thickness and breakdown ratio simultaneously; however, the configuration of this system is much simpler than that of the previous one. The simplification provides not only general versatility as an easy test method but also excellent reproducibility of the breakdown process of oil films. Furthermore, the method for calculating the film thickness and breakdown ratio has been improved by reconsidering an equivalent electrical circuit model. By using this novel experimental method, we aim to 
quantify the breakdown process of thin oil films induced by quasi-static loading and obtain basic knowledge about the transition from fluid film lubrication to boundary lubrication.

\section{Experimental details}

\subsection{Apparatus}

Figure 1 shows a schematic diagram of the principal part of the experimental apparatus. Basically, the apparatus employs a sample oil film formed between a steel ball and mercury loaded with its static pressure. This system is known as the steel-oil-mercury system.

The steel ball is made of SUJ2 and it is $15 \mathrm{~mm}$ in diameter. It is fixed to a holder with a magnetic force, and the holder is fixed to a tip of a horizontal double-cantilever spring with a stiffness of $1330 \mathrm{~N} / \mathrm{m}$. The spring is deflected when a reaction force is exerted by the static pressure of mercury. An optical displacement sensor measures the deflection of the spring for obtaining the load $W$. The spring, which is used for the vertical movement of the steel ball, is attached to a motorized stage along with some manual stages.

In the present study, the highest purity chemicals that were commercially available were employed. A stainless container contains mercury and sample oil. This container is placed on a thermostat plate with an insulating plate, which provides the accuracy of temperature in a range of $0.1^{\circ} \mathrm{C}$.

The steel ball and mercury are electrically connected to an impedance meter, which measures the complex impedance of the steel-oil-mercury system.

By using mercury as the counter material of steel, the surface of mercury is deformed along the steel surface, which is expected to create a single real contact area corresponding to an apparent contact area independently of shape, waviness, and roughness of the steel surface.

\subsection{Materials}

In the present study, we used the highest purity chemicals that were commercially available. Hexadecane was used as sample oil, which was percolated through a molecular sieve and filtered for eliminating a small amount of polar impurities ${ }^{(1)}$. For obtaining the purest form of mercury, it was stirred with acetone and separated with a funnel.

\subsection{Procedure}

At first, the steel ball and the stainless container were washed with acetone in an ultrasonic cleaner for $10 \mathrm{~min}$, dried in a thermostat chamber at $120{ }^{\circ} \mathrm{C}$ for $20 \mathrm{~min}$, and

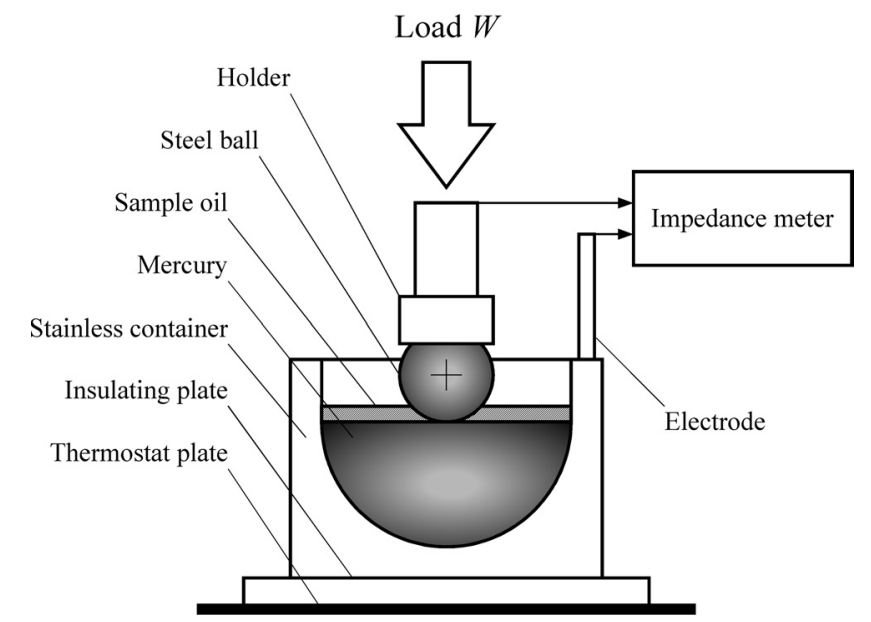

Fig. 1 Schematic diagram of the principal part of the experimental apparatus. 
cooled to room temperature for $20 \mathrm{~min}$. After their installation, 20-ml mercury was poured into the stainless container, and the temperature of the thermostat plate was maintained for $15 \mathrm{~min}$. Then, the steel ball was forced into mercury by a motorized stage with a constant indentation speed until the load reached to a setting value. The time evolutions of load and complex impedance of the system were measured for $200 \mathrm{~s}$ after the steel ball contacted the mercury surface.

After the measurement, 2-ml hexadecane was poured on mercury and maintained at the same setting temperature for $15 \mathrm{~min}$. The vertical position of the steel ball was adjusted for creating a gap of $1 \mathrm{~mm}$ between steel and mercury, and the same measurement was conducted. The typical indentation speed for realizing quasi-static loading was $40 \mu \mathrm{m} / \mathrm{s}$.

A digital data recorder stored the time evolutions of the load and complex impedance with a sampling frequency of $1 \mathrm{~Hz}$. An alternating voltage was applied for measuring the complex impedance with an effective voltage of $50 \mathrm{mV}$ and a frequency of $100 \mathrm{kHz}$.

\subsection{Derivation principles of film thickness and breakdown ratio}

A physical model of the present steel-oil-mercury system is shown in Fig. 2 (a). An oil film with a thickness $d$ exists between the steel and mercury surfaces. The total contact area is $S$, and the ratio of the intimate contact area to the total contact area, i.e., the breakdown ratio, is $\alpha$. Therefore, the area of the breakdown part is denoted by $\alpha S$ and that of the covered part is denoted by $(1-\alpha) S$.

An equivalent electrical circuit model is shown in Fig. 2 (b). The resistor $R_{1}$ represents the breakdown part, and the capacitor $C_{2}$ and resistor $R_{2}$ represent the covered part. In the present model, an external resistor $R_{0}$ is added to the previous one ${ }^{(4)}$ for further accuracy, which is determined by the measurement involving the direct contact between steel and mercury.

When an alternating voltage $V$ is applied to the steel-oil-mercury system, an alternating current $I$ passes through the circuit as

$$
\begin{aligned}
& V=|V| e^{j \omega t}, \\
& I=|I| e^{j(\omega t-\theta)} .
\end{aligned}
$$

Therefore, the complex impedance $Z$ is given by

$$
Z=V / I=|V / I| e^{j \theta}=|Z| e^{j \theta},
$$

where $j$ is the imaginary unit; $t$, the time; $\omega$, the angular frequency; and $\theta$, the phase difference. It should be noted that $Z$ includes two quantities, namely, the modulus $|Z|$ and argument $\theta$, as shown in Eq. (3).

On the other hand, from the equivalent electrical circuit model, $Z$ is expressed by using $R_{1}, C_{2}$, and $R_{2}$ as
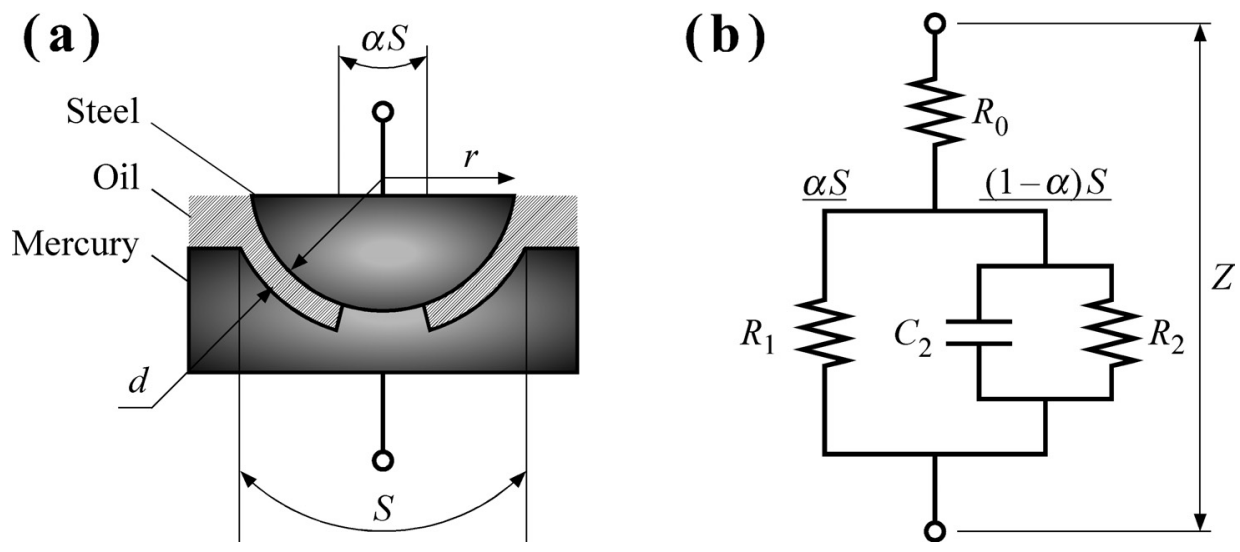

Fig. 2 Steel-oil-mercury system; (a) physical model and (b) electrical model. 


$$
Z=R_{0}+\left(R_{1}^{-1}+j \omega C_{2}+R_{2}^{-1}\right)^{-1} .
$$

In addition, $R_{1}, C_{2}$, and $R_{2}$ are given by

$$
\begin{aligned}
& R_{1}=R_{10} / \alpha, \\
& C_{2}=\varepsilon(1-\alpha) S / d, \\
& R_{2}=\gamma d /(1-\alpha) S,
\end{aligned}
$$

where $R_{10}$ is the electrical resistance when the oil film is broken perfectly ( $\left.\alpha=1\right)$, and $\varepsilon$ and $\gamma$ are the permittivity and resistivity of the oil film, respectively.

By using Eqs. (3)-(7), the film thickness $d$ and breakdown ratio $\alpha$ are given by

$$
\begin{aligned}
& d=-\frac{\varepsilon \omega(1-\alpha) S|Z|}{\sin \theta}\left\{\sin ^{2} \theta+\left(\cos \theta-\frac{R_{0}}{|Z|}\right)^{2}\right\}, \\
& \alpha=\frac{R_{10}}{|Z|}\left\{\frac{\sin \theta}{\gamma \varepsilon \omega}+\left(\cos \theta-\frac{R_{0}}{|Z|}\right)\right\}\left\{\sin ^{2} \theta+\left(\cos \theta-\frac{R_{0}}{|Z|}\right)^{2}\right\}^{-1} .
\end{aligned}
$$

From Eqs. (8) and (9), it can be seen that $d$ and $\alpha$ are obtained simultaneously if $Z$ is experimentally measured.

In addition, by solving Eqs. (3)-(7) for $\alpha=0$, we obtain

$\tan \theta_{1}=-\gamma \varepsilon \omega=$ const.

Equation (10) implies that $\theta_{1}$, which is measured experimentally, is independent of $d$ when the oil film is sufficiently thick.

In the present analysis, the following quantities were employed: $\varepsilon=2.05 \varepsilon_{0}[\mathrm{~F} / \mathrm{m}], \varepsilon_{0}=$ $8.85 \cdot 10^{-12}[\mathrm{~F} / \mathrm{m}]$ and $\theta_{1}=-89[\mathrm{deg}]$, where $\varepsilon_{0}$ is the electrical constant; the value of $\varepsilon$ was obtained from a literature for hexadecane ${ }^{(6)}$, which was assumed to be constant for the entire experiment. The other unknown quantities, i.e., $R_{0}$ and $R_{10}$, were determined as described later.

\section{Results and discussion}

\subsection{Contact between steel and mercury}

In order to obtain film thickness $d$ and breakdown ratio $\alpha$, we must quantify $R_{0}, R_{10}$, and $S$ included in Eqs. (8) and (9). For the determination of these values, the complex impedance was measured for the direct contact between steel and mercury without the sample oil.

Figure 3 shows the time evolutions of load $W$, the modulus of complex impedance $|Z|$, and the argument of the complex impedance $\theta$ under a temperature of $50{ }^{\circ} \mathrm{C}$, an indentation speed of $40 \mu \mathrm{m} / \mathrm{s}$, and a maximum load of $100 \mathrm{mN}$. In spite of the direct contact between steel and mercury, $\theta$ shows negative values; therefore, for the expression of the change in the complex impedance, a parallel circuit with a resistance and an unknown capacitance was introduced as an equivalent electrical model of the contact.

By considering the real part of the complex impedance, $R_{10}$ can be described as follows:

$$
R_{10}=|Z| \cos \theta-R_{0}+\frac{|Z|^{2} \sin ^{2} \theta}{|Z| \cos \theta-R_{0}} .
$$

It is inversely proportional to the contact area $S$ :

$$
R_{10} \propto S^{-1} \text {. }
$$

If it is assumed that $S$ is determined geometrically, it is obtained as a function of the indentation depth $h$ of the steel ball into mercury:

$$
S=2 \pi r h,
$$



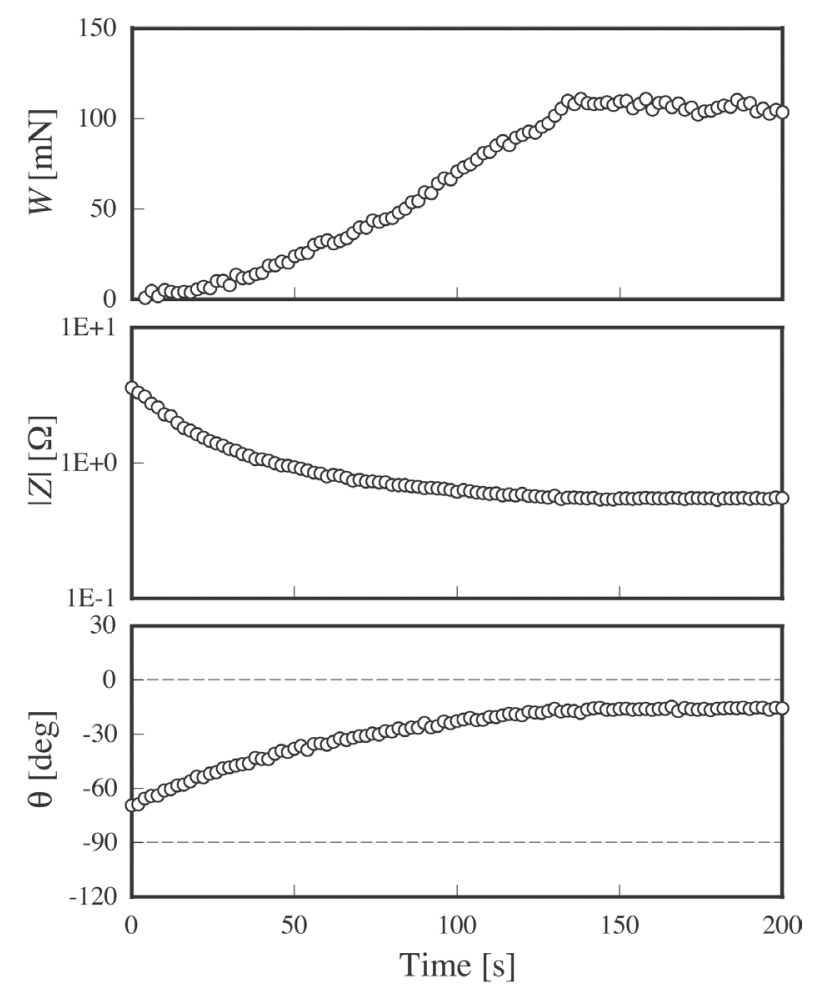

Fig. 3 Time evolutions of $W,|Z|$, and $\theta$ without sample oil; temperature of mercury: $50^{\circ} \mathrm{C}$, indentation speed: $40 \mu \mathrm{m} / \mathrm{s}$, maximum load: $100 \mathrm{mN}$.

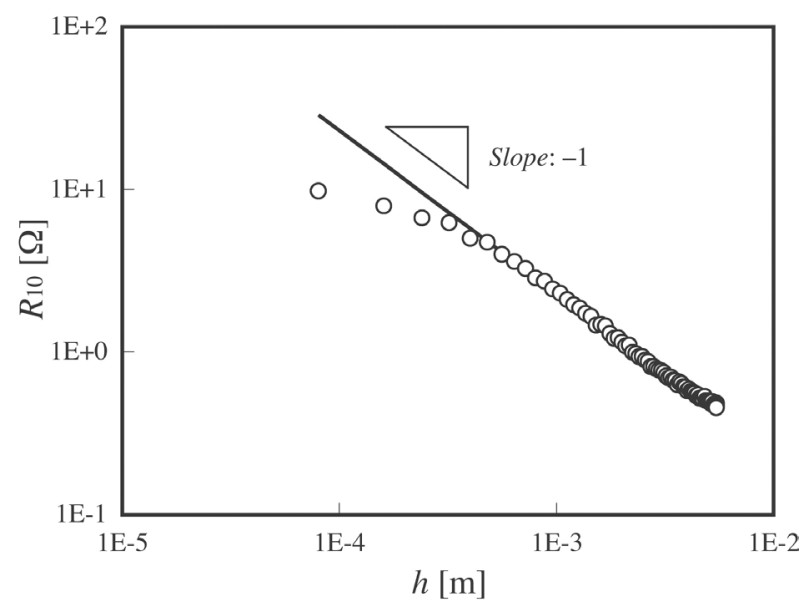

Fig. 4 Relationship between $R_{10}$ and $h$; open circle: $R_{10}$ obtained by Eq. (11), solid line: $R_{10}$ obtained by Eq. (14); $R_{0}=0.11[\Omega], \kappa=2.3 \cdot 10^{-3}[\Omega \cdot \mathrm{m}]$.

where $r$ is the radius of the steel ball. From Eqs. (12) and (13), the next relationship is obtained with a constant $\kappa$ :

$$
R_{10}=\kappa / h \text {. }
$$

Figure 4 shows the relationship between $R_{10}$ and $h$. The open circles denote $R_{10}$ determined by Eq. (11) with $|Z|$ and $\theta$ measured for direct contact between steel and mercury, and the solid line denotes $R_{10}$ determined by Eq. (14), where two unknown quantities $R_{0}$ and $\kappa$ are determined so that the open circles and solid line overlap well. In Fig. $4, R_{0}$ and $\kappa$ are $0.11 \Omega$ and $2.3 \cdot 10^{-3} \Omega \cdot \mathrm{m}$, respectively.

However, as shown in Fig. 4, the open circles deviate downward from the solid line when $h$ is smaller than approximately $0.5 \mathrm{~mm}$; this indicates that $S$ is not determined geometrically, as in Eq. (13), when $h$ is small. This must be the effect of the deformation of 


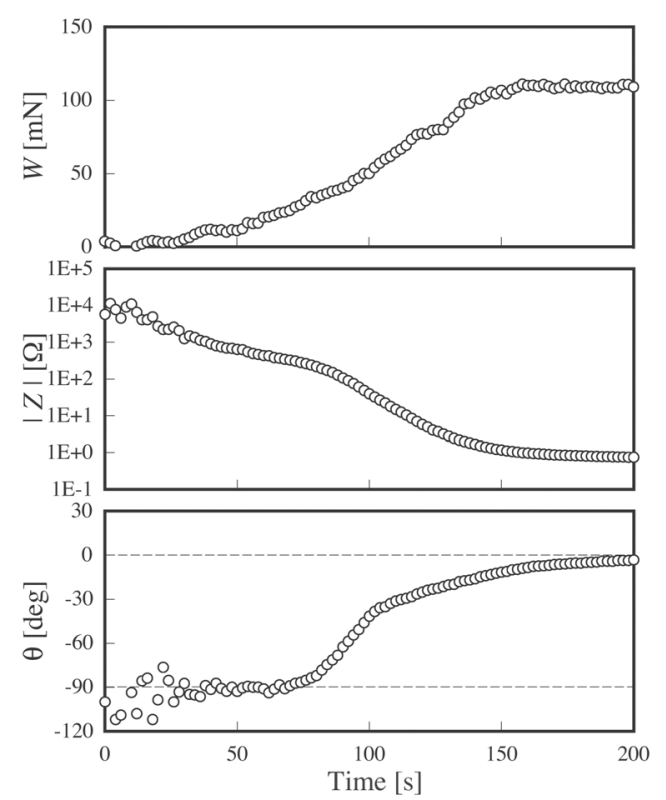

(a)

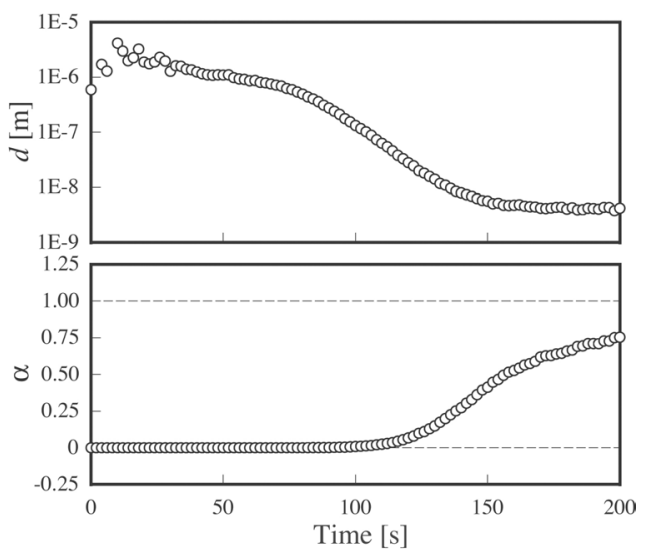

(b)

Fig. 5 Experimental results with hexadecane: (a) time evolutions of $W,|Z|$, and $\theta$ and (b) time evolutions of $d$ and $\alpha$; temperature of hexadecane: $50{ }^{\circ} \mathrm{C}$, indentation speed: $40 \mu \mathrm{m} / \mathrm{s}$, maximum load: $100 \mathrm{mN}$.

the mercury surface induced by its surface tension.

In the present study, the time evolution $S(t)$ was determined as

$S(t)=2 \pi r \kappa / R_{10}(t)$,

with the time evolution $R_{10}(t)$ obtained by Eq. (11). Assuming that the effect of the sample oil on the contact area $S$ was negligible, we used $S(t)$ and $R_{10}(t)$ for the determination of $d$ and $\alpha$ of the steel-oil-mercury system. This assumption could cause an error when $h$ is small, e.g., $h<0.5 \mathrm{~mm}$; however, it must be a good approximation when $h$ is large, e.g., $h>$ $0.5 \mathrm{~mm}$.

\subsection{Film thickness and breakdown ratio of hexadecane films}

Hexadecane was used as the sample oil. Figure 5 (a) shows the time evolutions of load $W$, the modulus of the complex impedance $|Z|$, and the argument of the complex impedance $\theta$ for a temperature of $50^{\circ} \mathrm{C}$, an indentation speed of $40 \mu \mathrm{m} / \mathrm{s}$, and a maximum load of 100 $\mathrm{mN}$. The obvious differences between Figs. 3 and 5 (a) are caused by the existence of hexadecane; this implies that the information about the hexadecane film should be included in Fig. 5 (a). In this case, it is found that $|Z|$ decreases and $\theta$ increases with $W$, and they approach asymptotic values.

Figure 5 (b) shows the time evolutions of film thickness $d$ and breakdown ratio $\alpha$, which are calculated by using Eqs. (8) and (9) based on the data in Fig. 5 (a). The thickness of the hexadecane film decreases due to loading; the minimum thickness is approximately 3 $\mathrm{nm}$ at $200 \mathrm{~s}$. On the other hand, $\alpha$ is zero for approximately $100 \mathrm{~s}$; thereafter, it gradually increases with the decrease of the film thickness; $\alpha$ is approximately 0.75 at $200 \mathrm{~s}$.

The film thickness obtained experimentally was compared with a hydrodynamic lubrication theory by assuming that the film thickness was homogeneous. According to this theory, the thickness $d$ of a squeezed film between parallel circular plates is given by ${ }^{(7)}$

$$
d=\left\{\left(\frac{4 W}{3 \eta r^{4}}\right) t+\frac{1}{d_{0}^{2}}\right\}^{-\frac{1}{2}},
$$

where $t$ is the time; $\eta$, the viscosity; $r$, the radius of the circular plate; $W$, the load; and $d_{0}$, the initial film thickness. 


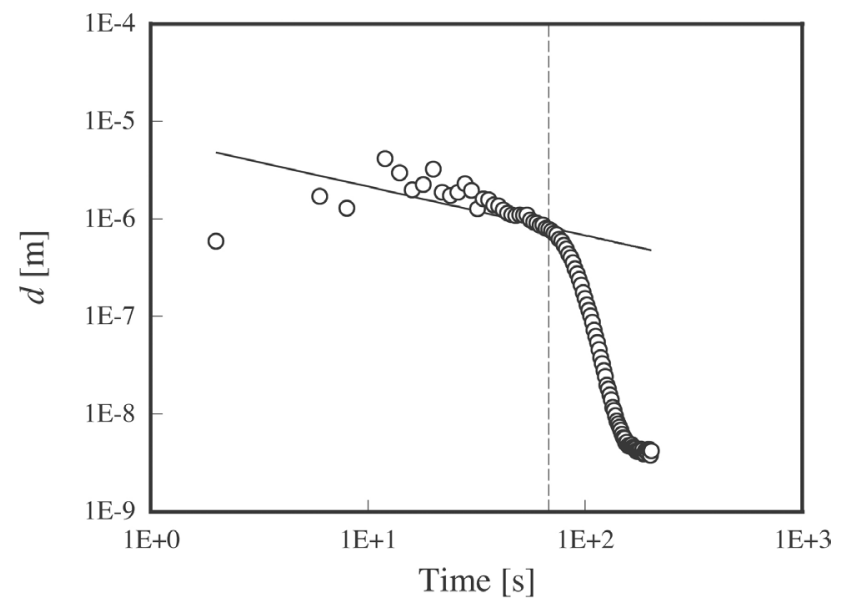

Fig. 6 Time evolution of film thickness $d$; open circle: experimental results shown in Fig. 5 (b), solid line: theoretical results with hydrodynamic lubrication obtained from Eq. (16).

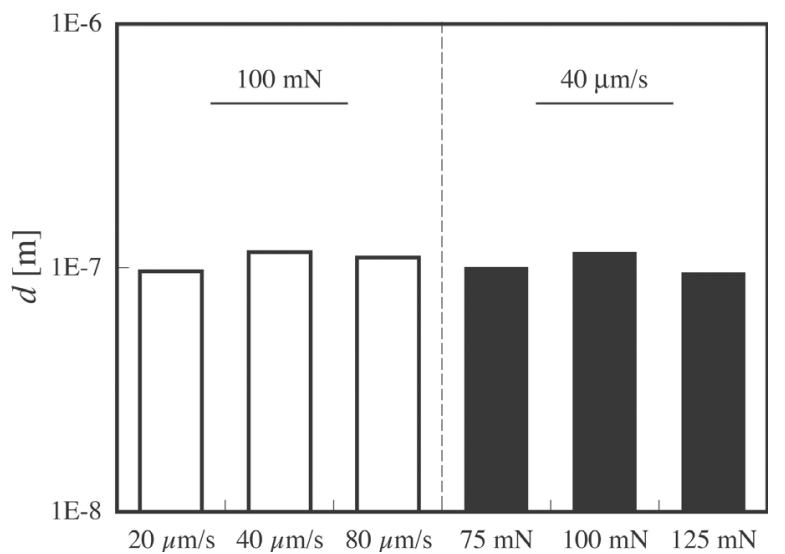

Fig. 7 Film thickness when a hexadecane film starts to break down; open bar: values obtained for a maximum load of $100 \mathrm{mN}$, solid bar: values obtained for a constant indentation speed of $40 \mu \mathrm{m} / \mathrm{s}$; temperature of hexadecane: $50{ }^{\circ} \mathrm{C}$.

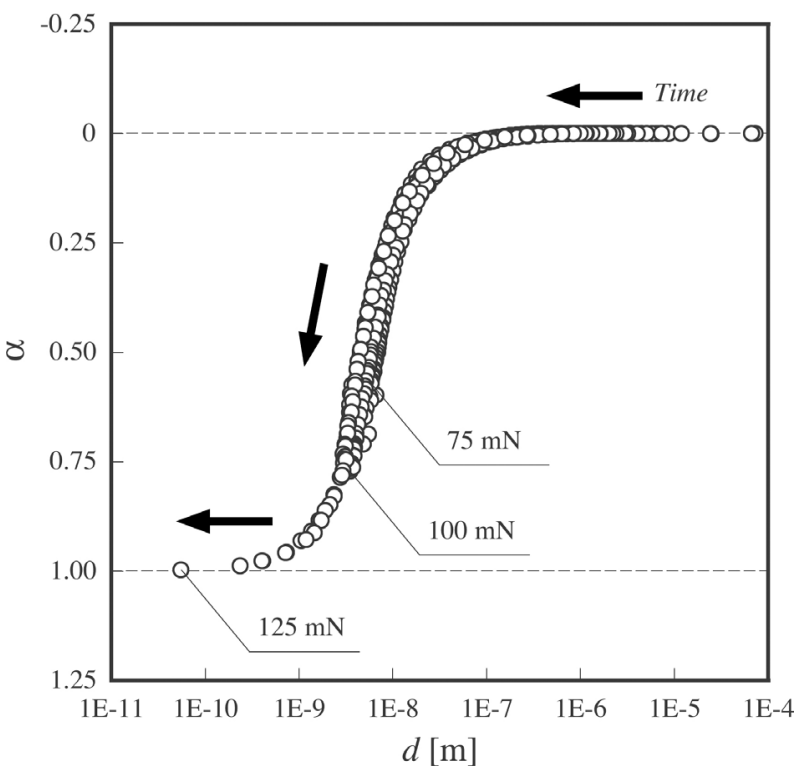

Fig. 8 Master curve of the breakdown processes of hexadecane films; temperature of hexadecane: $50{ }^{\circ} \mathrm{C}$. 
Figure 6 shows the time evolution of the film thickness with a double-logarithmic plot, where the open circles denote the experimental result, and the solid line denotes the theoretical result obtained using Eq. (16). When the film is thicker than approximately 1 $\mu \mathrm{m}$, it appears that the experimental result is in good agreement with the theoretical one. On the other hand, when the film is thinner than approximately $1 \mu \mathrm{m}$, the experimental result deviates significantly from the theoretical one.

\subsection{Breakdown initiation and progress of oil films}

Figure 7 shows film thickness $d$, when the hexadecane film starts to break down, i.e., $\alpha=0.01$, for five types of experimental conditions; the open bars show the result obtained for a maximum load of $100 \mathrm{mN}$, and the solid bars show the result obtained for a indentation speed of $40 \mu \mathrm{m} / \mathrm{s}$. It appears that the film thickness at the breakdown initiation is constant, i.e., approximately $100 \mathrm{~nm}$, independently of the indentation speed and maximum load.

In general, it is believed that the initiation of oil-film breakdown depends on the surface roughness as the film parameter $\Lambda$ is used for its estimation. However, the effect of surface roughness is minimized in the present system by using the fluidity of mercury; therefore, the initiation of the oil-film breakdown should be independent of experimental conditions.

Figure 8 shows a plot of breakdown ratio $\alpha$ against film thickness $d$ for the five different conditions shown in Fig. 7. It is found that all the data points create a single curve, which should be called the master curve of the breakdown process of hexadecane films. The path of the breakdown on the master curve does not depend on the indentation speed, and the maximum load determines the final point of the breakdown process.

From the master curve, it is concluded that the breakdown of hexadecane films occurs in two stages: one is the decrement of the thickness without breakdown, i.e., $d>100 \mathrm{~nm}$, and the other is the decrement of the thickness with the drastic progress of breakdown, i.e., $d<100 \mathrm{~nm}$.

\section{Conclusions}

In the present study, the thickness and breakdown ratio of hexadecane films have been simultaneously measured by using a steel-oil-mercury system along with complex impedance analysis. The breakdown process of these films induced by quasi-static loading has also been investigated.

(1) The present system realizes the simultaneous measurement of thickness and breakdown ratio of an oil film under quasi-static loading. The minimum film thickness measurable by the system is at least a nanometer level.

(2) The relationship between the thickness and the breakdown ratio of hexadecane films is represented by a single master curve. Every breakdown process traces on the master curve and a load determines the final point.

(3) The breakdown of hexadecane films occurs in two stages: one is the decrement of the thickness without breakdown, i.e., $d>100 \mathrm{~nm}$, and the other is the decrement of the thickness with the drastic progress of breakdown, i.e., $d<100 \mathrm{~nm}$.

\section{Acknowledgement}

The authors would like to express their sincere gratitude to Nippon Oil Corporation for their helpful discussion as well as financial support. 


\section{References}

(1) Smith, A. J. \& Cameron, A., Rigid Surface Films, Proceedings of the Royal Society of London, Series A, Vol.328, No.1575 (1972), pp.541-560.

(2) Stribeck, R., Die Wesentlichen Eigenschaften der Gleit-und Rollenlager, Zeitschrift des Vereins Deutscher Ingenieure, Vol.46, No.36 (1902), pp.1341-1348.

(3) Jacobson, B., The Stribeck Memorial Lecture, Tribology International, Vol.36, No.11 (2003), pp.781-789.

(4) Nakano, K. \& Akiyama, Y., Simultaneous Measurement of Film Thickness and Coverage of Loaded Boundary Films with Complex Impedance Analysis, Tribology Letters, Vol.22, No.1 (2006), pp.127-134.

(5) Bowden, F. P. \& Tabor, D., The Friction and Lubrication of Solids (Oxford University Press, London, 1950).

(6) Abboud, J. -L. M. \& Notario, R., Critical Compilation of Scales of Solvent Parameter. Part I. Pure, None-Hydrogen Bond Donor Solvents, Pure and Applied Chemistry, Vol.71, No.4 (1999), pp.645-718.

(7) Cameron, A., Principles of Lubrication (Longmans, London, 1966). 